Research Paper

\title{
HMGBI genetic polymorphisms are biomarkers for the development and progression of breast cancer
}

\author{
Bi-Fei Huang1\#, Huey-En Tzeng2,3,4\#, Po-Chun Chen5 ${ }^{1}$ Chao-Qun Wang1', Chen-Ming Su6, Yan Wang7, \\ Gui-Nv Hu${ }^{8}$, Yong-Ming Zhao ${ }^{8}$, Qian Wang ${ }^{1}$, Chih-Hsin Tang, $9,5,10 \bowtie$ \\ 1. Department of Pathology, Affiliated Dongyang Hospital of Wenzhou Medical University, Dongyang, Zhejiang, China \\ 2. Taipei Cancer Center, Taipei Medical University, Taipei, Taiwan \\ 3. Graduate Institute of Cancer Biology and Drug Discovery, College of Medical Science and Technology, Taipei Medical University, Taipei, Taiwan \\ 4. Department of Internal Medicine, Division of Hematology and Oncology, Taipei Medical University Hospital, Taipei, Taiwan \\ 5. Graduate Institute of Biomedical Science, China Medical University, Taichung, Taiwan \\ 6. Laboratory of Biomedicine, Affiliated Dongyang Hospital of Wenzhou Medical University, Dongyang, Zhejiang, China \\ 7. Department of Medical Oncology, Affiliated Dongyang Hospital of Wenzhou Medical University, Dongyang, Zhejiang, China \\ 8. Department of Surgical Oncology, Affiliated Dongyang Hospital of Wenzhou Medical University, Dongyang, Zhejiang, China \\ 9. Department of Pharmacology, School of Medicine, China Medical University, Taichung, Taiwan \\ 10. Department of Biotechnology, College of Health Science, Asia University, Taichung, Taiwan \\ \# These authors have contributed equally to this work \\ $\triangle$ Corresponding author: Chih-Hsin Tang PhD, Department of Pharmacology, School of Medicine, China Medical University, Taichung, Taiwan; E-mail: \\ chtang@mail.cmu.edu.tw \\ (1) Ivyspring International Publisher. This is an open access article distributed under the terms of the Creative Commons Attribution (CC BY-NC) license \\ (https://creativecommons.org/licenses/by-nc/4.0/). See http://ivyspring.com/terms for full terms and conditions.
}

Received: 2017.10.23; Accepted: 2018.03.01; Published: 2018.03.12

\begin{abstract}
Breast cancer is a major cause of cancer mortality worldwide. High-mobility group box protein 1 (HMGBI) is a ubiquitous nuclear protein found in all mammal eukaryotic cells that participates in tumor progression, migration and metastasis. HMGBI overexpression has been indicated in breast cancer patients. However, scant information is available regarding the association between HMGBI single nucleotide polymorphisms (SNPs) and the risk or prognosis of breast cancer. We report on the association between 4 SNPs of the HMGBI gene (rs1360485, rs1045411, rs 2249825 and rs 1412125) and breast cancer susceptibility as well as clinical outcomes in 313 patients with breast cancer and in 217 healthy controls. Patients with one G allele in the rs 1360485 or rs2249825 domains are likely to progress to T2 tumor and lymph node metastasis. In addition, the presence of one G allele in SNPs rs 1360485 or rs 2249825 was associated with a higher risk of progressing to T2 tumor and distant metastasis amongst HER2-enriched and triple-negative breast cancer (TNBC) tumors compared with luminal $A$ and luminal $B$ tumors. Furthermore, having one $C$ allele in the rs 1412125 domain increased the risk of pathologic grade 3 disease in HER2-enriched and TNBC tumors. Our results indicate that genetic variations in the HMGBI gene may serve as an important predictor of breast cancer progression and metastasis.
\end{abstract}

Key words: HMGB1 polymorphisms; Breast cancer; Single nucleotide polymorphism; Susceptibility

\section{Introduction}

Breast cancer is associated with high mortality. Over a million women worldwide are diagnosed with breast cancer every year and over 500,000 succumb to the disease [1]. Risk factors associated with breast cancer in women include age, family history, reproductive and gynecologic factors, as well as lifestyle factors such as alcohol consumption and lack of physical activity, amongst others [2]. Women who are at high risk of breast cancer may be advised to maintain their mammography screening schedule, undergo genetic testing, or commence chemoprevention.

Current statistical models for estimating breast cancer risk have limited sensitivity and specificity [2]. Researchers have therefore explored genetic variation associated with breast cancer risk, in order to 
determine whether single nucleotide polymorphism (SNP) genotyping will more accurately stratify breast cancer risk and guide disease management. Emerging reports indicate an association between SNPs in certain genes and susceptibility to breast cancer, as well as clinicopathologic status. Besides the recognized $B R C A 1$ and $B R C A 2$ mutations that markedly increase the risk of developing breast cancer $[3,4]$, a number of additional low- and moderate-risk susceptibility variants have been identified, including caspase-8 (CASP8), an enzyme involved in apoptosis [5].

High-mobility group box protein 1 (HMGB1) is a ubiquitous nuclear protein that has been discovered in mammals [6, 7]. HMGB1 contains DNA binding domains and contributes to DNA repair and the stabilization of nuclear homeostasis [8]. HMGB1 is usually localized in the cell nucleus and is secreted into the extracellular environment in response to different stimuli; either passively during cellular apoptosis or necrosis, or actively following inflammatory signals from activated immune cells or neuronal cells [9]. It has been reported HMGB1 SNPs controls with rheumatoid arthritis disease outcome [10]. Previous research has confirmed the association of HMGB1 SNPs with the susceptibility and progression of disease, such as hepatocellular carcinoma [11], lung cancer [12] and uterine cervical neoplasia [13]. An increase in HMGB1 levels in response to neoadjuvant chemotherapy has been found to be a prognostic marker of survival in early breast cancer patients [14] and recent research has demonstrated a cumulative impact of multiple risk-associated polymorphisms in the HMGB1/receptor for advanced glycation end products (HMGB1/RAGE) pathway upon breast cancer progression [15]. However, the association between HMGB1 SNPs and breast cancer risk, prognosis, metastasis and clinical aspects is unclear. We therefore conducted a case-control study to evaluate the role of HMGB1 SNPs in breast cancer susceptibility and clinicopathologic features in a cohort of Chinese Han individuals.

\section{Materials and Methods}

\section{Participants}

Between 2014 and 2016, we collected 313 blood specimens from patients (cases) who had been diagnosed with breast cancer at Dongyang People's Hospital. The control group consisted of 217 healthy participants without a history of cancer. All participants provided written informed consent, and the study was approved by the Ethics Committee of Dongyang People's Hospital. Pathohistologic diagnosis followed the World Health Organization classification of breast tumors and tumors were graded using the Scarff-Bloom-Richardson method [16]. Breast cancer cases were categorized by estrogen receptor (ER), progesterone receptor (PR), human epidermal growth factor receptor 2 (HER2) and Ki-67

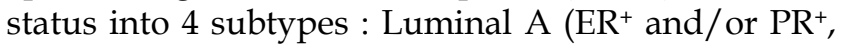
HER2-, Ki-67 <14\%); Luminal B (ER ${ }^{+}$and/or $\mathrm{PR}^{+}$, HER2-, Ki-67 $\geq 14 \%$; or $\mathrm{ER}^{+}$and/or $\left.\mathrm{PR}^{+}, \mathrm{HER}^{+}\right)$; HER2-enriched (ER $\left.{ }^{-}, \mathrm{PR}^{-}, \mathrm{HER} 2^{+}\right)$; or TNBC (ER-, PR $^{-}$, HER2 ${ }^{-}$) [17-19]. Demographic data on age, sex, smoking history and alcohol consumption were obtained from a standardized questionnaire and electronic medical records.

\section{SNP selection}

SNP rs2249825 (3814C/G; genomic number $31,037,903$ ) near the exon, rs1360485 (3'UTR, T/C; genomic number $31,031,884)$ in the $3^{\prime}$ untranslated region, SNP rs1412125 (-1615T/C; genomic number $31,041,595)$ in the promoter region and rs1045411 $(2262 \mathrm{C} / \mathrm{T}$; genomic number $31,033,232)$ in the exon were selected according to Chinese HapMap data and previous studies $[13,20]$. The minor allele frequencies of these SNPs were all $\geq 5 \%$.

\section{Determination of genotypes}

Total genomic DNA was isolated from whole blood specimens using QIAamp DNA blood mini kits (Qiagen, Valencia, CA), as per the manufacturer's instructions. DNA was dissolved in a Tris-EDTA (TE) buffer containing $10 \mathrm{mM}$ Tris-HCl, $1 \mathrm{mM}$ EDTA Na ( $\mathrm{pH} 7.8$ ) and stored at $-20^{\circ} \mathrm{C}$ until it was subjected to quantitative polymerase chain reaction (PCR) analysis. Four HMGB1 SNPs (rs1360485, rs1045411, rs2249825 and rs1412125) were examined with the use of a commercially available TaqMan SNP genotyping assay (Applied Biosystems, Warrington, UK), according to the manufacturer's protocols [21, 22].

\section{Statistical analysis}

The genotype distribution of each SNP was analyzed for Hardy-Weinberg equilibrium and confirmed by Chi-square analysis. Demographic characteristics were compared between patients and controls using the Mann-Whitney U-test and Fisher's exact test. Associations between genotypes, breast cancer risk and clinicopathologic characteristics were estimated using adjusted odds ratios (AORs) and 95\% confidence intervals (CIs), after controlling for other covariates. Significant differences in haplotype frequencies between cases and controls were analyzed using Haploview, according to the software package [23]. A $p$ value of $<0.05$ was considered statistically significant. Data were analyzed using SAS statistical software (Version 9.1, 2005; SAS Institute Inc., Cary, NC). 


\section{Results}

Sociodemographic characteristics and clinical parameters for all study participants are shown in Table 1. Significant between-group differences were observed for age, tobacco use and alcohol consumption $(p<0.05)$. Most patients $(76.7 \%)$ had stage I/II breast cancer; $23.3 \%$ had stage III/IV disease (Table 1 ). In addition, the majority of patients were ER-negative $(69.6 \%)$ or HER2-positive $(63.6 \%)$ (Table 1$)$.

Table 1. Baseline demographic and clinical characteristics of the study population.

\begin{tabular}{|c|c|c|c|}
\hline Variable & $\begin{array}{l}\text { Controls }(n=217) \\
\text { N }(\%)\end{array}$ & $\begin{array}{l}\text { Patients }(n=313) \\
\text { N (\%) }\end{array}$ & $p$ value \\
\hline Age (years) & $\begin{array}{l}\text { Mean } \pm \text { S.D. } \\
43.4 \pm 17.1\end{array}$ & $\begin{array}{l}\text { Mean } \pm \text { S.D. } \\
53.2 \pm 11.4\end{array}$ & $<0.001^{*}$ \\
\hline \multicolumn{4}{|c|}{ Alcohol consumption } \\
\hline No & $176(81.1)$ & $294(93.9)$ & $<0.05$ \\
\hline Yes & $41(18.9)$ & $19(6.1)$ & \\
\hline \multicolumn{4}{|c|}{ Tobacco consumption } \\
\hline No & $187(86.2)$ & $311(99.4)$ & $<0.05$ \\
\hline Yes & $30(13.8)$ & $2(0.6)$ & \\
\hline \multicolumn{4}{|c|}{ Clinical stage } \\
\hline I-II & & $240(76.7)$ & \\
\hline III-IV & & $73(23.3)$ & \\
\hline \multicolumn{4}{|c|}{ Tumor T status } \\
\hline$\leq \mathrm{T} 2$ & & $297(94.9)$ & \\
\hline$>\mathrm{T} 2$ & & $16(5.1)$ & \\
\hline \multicolumn{4}{|c|}{ Lymph node status } \\
\hline No & & $160(51.1)$ & \\
\hline$>\mathrm{N} 0$ & & $153(48.9)$ & \\
\hline \multicolumn{4}{|c|}{ Distant metastasis } \\
\hline M0 & & $303(96.8)$ & \\
\hline M1 & & $10(3.2)$ & \\
\hline \multicolumn{4}{|c|}{ Histologic grade } \\
\hline $\mathrm{G} 1+\mathrm{G} 2$ & & $187(59.7)$ & \\
\hline G3 & & $125(39.9)$ & \\
\hline \multicolumn{4}{|l|}{ ER status } \\
\hline Positive & & $95(30.4)$ & \\
\hline Negative & & $218(69.6)$ & \\
\hline \multicolumn{4}{|l|}{ PR status } \\
\hline Positive & & $144(46)$ & \\
\hline Negative & & $169(54)$ & \\
\hline \multicolumn{4}{|l|}{ HER2 } \\
\hline Positive & & $199(63.6)$ & \\
\hline Negative & & $114(36.4)$ & \\
\hline
\end{tabular}

S.D. $=$ standard deviation; $\mathrm{T}=$ primary tumor; $\mathrm{T} 1=$ tumor $\leq 5 \mathrm{~cm} ; \mathrm{T} 2=$ tumor $>5 \mathrm{~cm} ; \mathrm{N} 0=$ no regional lymph node metastasis; $\mathrm{M} 0=$ no clinical or radiographic evidence of distant metastasis; M1 = distant detectable metastases as determined by classic clinical and radiographic means and/or histologically proven $>0.2 \mathrm{~mm} ; \mathrm{G} 1=$ well differentiated; $\mathrm{G} 2=$ moderately differentiate; $\mathrm{G} 3$ = poorly differentiated; $\mathrm{ER}=$ estrogen receptor; $\mathrm{PR}=$ progesterone receptor; HER2 = human epidermal growth factor receptor 2 .

The Mann-Whitney U test or Fisher's exact test was used to compare values between controls and patients with breast cancer. ${ }^{*} p$ value $<0.05$ was considered statistically significant.

HMGB1 genotype distribution patterns for all participants are shown in Table 2. In the healthy controls, all genotypic frequencies were in Hardy-Weinberg equilibrium $(p>0.05)$. In both patients and controls, most of those with the rs1360485, rs1045411, rs2249825 and rs1412125 SNPs were, respectively, homozygous for $\mathrm{A} / \mathrm{A}$, homozygous for $\mathrm{G} / \mathrm{G}$, homozygous for $\mathrm{C} / \mathrm{C}$, and homozygous for $\mathrm{T} / \mathrm{T}$ alleles (Table 2 ). In analyses adjusted for potential confounders, there were no significant differences between cases and controls in regard to the frequency of each of the 4 studied polymorphisms (Table 2 ).

Next, we compared the distributions of clinical aspects and HMGB1 genotypes amongst cases. We found that patients with one $G$ allele in the rs1360485 SNP (AOR 2.466; 95\% CI: 1.068-5.694), one G allele in the rs2249825 SNP (AOR 3.264; 95\% CI: 1.330-8.011), or one C allele in the rs1412125 SNP (AOR 2.702; 95\% CI: 1.181-6.182) were more likely to progress to T2 breast cancer (Table 3). Patients with one G allele in the rs1360485 SNP (AOR 1.444; 95\% CI: 0.944-2.207), one A allele in the rs1045411 (AOR 1.443; 95\% CI: $0.935-2.228$, or one $G$ allele in the rs2249825 (AOR 1.515; 95\% CI: 0.937-2.448) were at increased risk of developing lymph node metastasis disease $(\mathrm{N} 2+\mathrm{N} 3)$ (Table 3).

In an analysis of clinical aspects and HMGB1 genotypic frequencies in different breast cancer subtypes, we found no significant differences between cases and controls in regard to the frequency of HMGB1 polymorphisms (Table 4).

In HER2 and TNBC subtypes, patients with one $\mathrm{G}$ allele in the rs1360485 SNP (AOR 6.061; 95\% CI: 2.190-16.774), one A allele in the rs1045411 SNP (AOR 3.321; 95\% CI: 1.216-9.068), one $G$ allele in the rs2249825 SNP (AOR 5.800; 95\% CI: 2.098-16.033), or one C allele in the rs1412125 SNP (AOR 5.849; 95\% CI: 2.116-16.165) were likely to progress to $\mathrm{T} 2$ breast cancer (Table 5). Individuals with one $\mathrm{G}$ allele in the rs1360485 SNP (AOR 4.918; 95\% CI: 1.479-16.353), or one A allele in the rs1045411 SNP (OR 5.847; 95\% CI: 1.749-19.551) were likely to progress to distant metastatic disease (Table 5). Furthermore, the presence of one $C$ allele in the rs1412125 SNP (AOR 2.112; 95\% CI: 1.028-4.341) increased the likelihood of developing pathologic grade (G3) disease (Table 5).

\section{Discussion}

HMGB1 plays multiple roles inside and outside cells, such as chromatin stabilization, DNA repair, gene transcription, program cell death regulation, and immune response. The HMGB1 gene has been implicated in tumor progression in various types of cancer such as colon, liver, breast, oral, and lung cancer [11, 24-26]. Previous research has indicated that HMGB1 plays a role in breast cancer progression and metastasis [14, 27] and that inhibiting HMGB1 expression with quercetin promotes apoptosis in human breast adenocarcinoma cells [28]. These results suggest that HMGB1 knockdown might be a valuable therapeutic strategy for breast cancer. 
Table 2. Distribution frequencies of $H M G B I$ genotypes and 4 SNP alleles in controls and patients with breast cancer.

\begin{tabular}{|c|c|c|c|c|c|c|}
\hline Variable & $\begin{array}{l}\text { Controls }(\mathrm{n}=217) \\
\mathrm{N}(\%)\end{array}$ & $\begin{array}{l}\text { Patients }(n=313) \\
\text { N }(\%)\end{array}$ & OR $(95 \%$ CI) & $p$ value & $\operatorname{AOR}^{a}(95 \% \mathrm{CI})$ & $p$ value \\
\hline \multicolumn{7}{|c|}{ rs1360485 } \\
\hline AA & $131(60.4)$ & $191(61.0)$ & 1.00 (reference) & & 1.00 (reference) & \\
\hline AG & $71(32.7)$ & 99 (31.6) & $0.956(0.656-1.395)$ & 0.82 & $0.947(0.636-1.412)$ & 0.79 \\
\hline GG & $15(6.9)$ & $23(7.3)$ & $1.052(0.529-2.091)$ & 0.89 & $1.020(0.496-2.098)$ & 0.96 \\
\hline AA & $131(60.4)$ & $191(61.0)$ & 1.00 (reference) & & 1.00 (reference) & \\
\hline $\mathrm{AG}+\mathrm{GG}$ & $86(39.6)$ & $122(39.0)$ & $0.973(0.683-1.387)$ & 0.88 & $0.949(0.654-1.378)$ & 0.78 \\
\hline A & $333(76.7)$ & $481(76.8)$ & 1.00 (reference) & & 1.00 (reference) & \\
\hline G & $101(23.3)$ & 145 (23.2) & $0.994(0.744-1.328)$ & 0.97 & $0.995(0.664-1.491)$ & 0.98 \\
\hline \multicolumn{7}{|l|}{ rs1045411 } \\
\hline GG & $132(60.8)$ & $200(63.9)$ & 1.00 (reference) & & 1.00 (reference) & \\
\hline GA & 75 (34.6) & $90(28.8)$ & $0.792(0.543-1.155)$ & 0.23 & $0.763(0.513-1.135)$ & 0.18 \\
\hline AA & $10(4.6)$ & $23(7.3)$ & $1.518(0.700-3.293)$ & 0.29 & $1.551(0.677-3.558)$ & 0.3 \\
\hline GG & $132(60.8)$ & $200(63.9)$ & 1.00 (reference) & & 1.00 (reference) & \\
\hline $\mathrm{GA}+\mathrm{AA}$ & $85(39.2)$ & $113(36.1)$ & $0.877(0.614-1.254)$ & 0.47 & $0.845(0.581-1.230)$ & 0.38 \\
\hline G & $339(78.1)$ & $490(78.3)$ & 1.00 (reference) & & 1.00 (reference) & \\
\hline A & $95(21.9)$ & $136(21.7)$ & $0.990(0.736-1.332)$ & 0.95 & $1.082(0.708-1.653)$ & 0.72 \\
\hline \multicolumn{7}{|l|}{ rs2249825 } \\
\hline $\mathrm{CC}$ & $163(75.1)$ & $214(68.4)$ & 1.00 (reference) & & 1.00 (reference) & \\
\hline CG & $48(22.1)$ & $91(29.1)$ & $1.444(0.963-2.164)$ & 0.07 & $1.354(0.885-2.070)$ & 0.16 \\
\hline GG & $6(2.8)$ & $8(2.6)$ & $1.016(0.346-2.984)$ & 0.98 & $1.015(0.323-3.189)$ & 0.98 \\
\hline $\mathrm{CC}$ & $163(75.1)$ & $214(68.4)$ & 1.00 (reference) & & 1.00 (reference) & \\
\hline $\mathrm{CG}+\mathrm{GG}$ & $54(24.9)$ & 99 (31.6) & $1.396(0.946-2.061)$ & 0.09 & $1.313(0.873-1.977)$ & 0.19 \\
\hline C & $374(86.2)$ & $519(82.9)$ & 1.00 (reference) & & 1.00 (reference) & \\
\hline G & $60(13.8)$ & $107(17.1)$ & $1.285(0.912-1.811)$ & 0.15 & 1.177 (0.737-1.879) & 0.5 \\
\hline \multicolumn{7}{|l|}{ rs1412125 } \\
\hline TT & $132(60.8)$ & $170(54.3)$ & 1.00 (reference) & & 1.00 (reference) & \\
\hline TC & $70(32.3)$ & $122(39.0)$ & $1.353(0.933-1.962)$ & 0.11 & $1.306(0.884-1.931)$ & 0.18 \\
\hline $\mathrm{CC}$ & $15(6.9)$ & $21(6.7)$ & $1.087(0.540-2.190)$ & 0.82 & $1.131(0.533-2.398)$ & 0.75 \\
\hline TT & $132(60.8)$ & $170(54.3)$ & 1.00 (reference) & & 1.00 (reference) & \\
\hline $\mathrm{TC}+\mathrm{CC}$ & $85(39.2)$ & $143(45.7)$ & $1.306(0.919-1.857)$ & 0.14 & $1.266(0.873-1.835)$ & 0.21 \\
\hline $\mathrm{T}$ & $334(77)$ & $462(73.8)$ & 1.00 (reference) & & 1.00 (reference) & \\
\hline C & $100(23)$ & $164(26.2)$ & $1.186(0.891-1.578)$ & 0.24 & $1.267(0.851-1.885)$ & 0.24 \\
\hline
\end{tabular}

$\mathrm{OR}=$ odds ratio; $\mathrm{AOR}=$ adjusted odds ratio; $\mathrm{CI}=$ confidence interval.

a Logistic regression analysis adjusted for age, tobacco and alcohol consumption.

Table 3. Association of $H M G B I$ alleles and 4 SNPs with the development and progression of breast cancer.

\begin{tabular}{|c|c|c|c|c|c|c|c|c|c|c|}
\hline \multirow[t]{3}{*}{ Allele } & \multicolumn{10}{|c|}{ Patients $(n=626) N(\%)$} \\
\hline & \multicolumn{2}{|c|}{ Clinical stage } & \multicolumn{2}{|c|}{ Tumor size } & \multicolumn{2}{|c|}{ Lymph node metastasis } & \multicolumn{2}{|c|}{ Distant metastasis } & \multicolumn{2}{|c|}{ Pathologic grade } \\
\hline & Stage I/II & Stage III/IV & $\leqq \mathrm{T} 2$ & $>\mathrm{T} 2$ & N0+N1 & $\mathrm{N} 2+\mathrm{N} 3$ & M0 & M1 & G1+G2 & G3 \\
\hline \multicolumn{11}{|l|}{ rs1360485 } \\
\hline A & $366(76.1)$ & $114(78.6)$ & $462(96.0)$ & $132(91.0)$ & $250(52.0)$ & $70(48.3)$ & $469(97.5)$ & $137(94.5)$ & $333(69.5)$ & $99(68.3)$ \\
\hline G & $115(23.9)$ & $31(21.4)$ & $19(4.0)$ & $13(9.0)$ & $231(48.0)$ & 75 (51.7) & $12(2.5)$ & $8(5.5)$ & $146(30.5)$ & $46(31.7)$ \\
\hline OR $(95 \% \mathrm{CI})$ & 1 & $\begin{array}{l}0.865 \\
(0.552-1.356)\end{array}$ & 1.00 & $\begin{array}{l}2.395 \\
(1.152-4.977)^{*}\end{array}$ & 1.00 & $\begin{array}{l}1.160 \\
(0.800-1.681)\end{array}$ & 1.00 & $\begin{array}{l}2.282 \\
(0.914-5.696)\end{array}$ & 1.00 & $\begin{array}{l}1.060 \\
(0.710-1.581)\end{array}$ \\
\hline $\operatorname{AOR}(95 \% \mathrm{CI})^{\mathrm{a}}$ & 1 & $\begin{array}{l}0.861 \\
(0.513-1.446)\end{array}$ & 1.00 & $\begin{array}{l}2.466 \\
(1.068-5.694)^{*}\end{array}$ & 1.00 & $\begin{array}{l}1.444 \\
(0.944-2.207)^{*}\end{array}$ & 1.00 & $\begin{array}{l}2.480 \\
(0.824-7.458)\end{array}$ & 1.00 & $\begin{array}{l}0.746 \\
(0.464-1.199)\end{array}$ \\
\hline \multicolumn{11}{|l|}{ rs1045411 } \\
\hline G & $369(75.3)$ & $111(81.6)$ & $467(95.3)$ & $127(93.4)$ & $254(51.8)$ & $66(48 ; 5)$ & $477(97.3)$ & $129(94.9)$ & $337(69.1)$ & 95 (69.9) \\
\hline A & $121(24.7)$ & $25(18.4)$ & $23(4.7)$ & $9(6.6)$ & $236(48.2)$ & $70(51.5)$ & $13(2.7)$ & $7(5.1)$ & $151(30.9)$ & $41(30.1)$ \\
\hline OR $(95 \% \mathrm{CI})$ & 1 & $\begin{array}{l}0.687 \\
(0.425-1.110)\end{array}$ & 1.00 & $\begin{array}{l}1.439 \\
(0.650-3.187)\end{array}$ & 1.00 & $\begin{array}{l}1.141 \\
(0.781-1.669)\end{array}$ & 1.00 & $\begin{array}{l}1.991 \\
(0.778-5.093)\end{array}$ & 1.00 & $\begin{array}{l}0.963 \\
(0.637-1.456)\end{array}$ \\
\hline $\operatorname{AOR}(95 \% \mathrm{CI})$ & 1 & $\begin{array}{l}0.704 \\
(0.406-1.221)\end{array}$ & 1.00 & $\begin{array}{l}1.521 \\
(0.625-3.700)\end{array}$ & 1.00 & $\begin{array}{l}1.443 \\
(0.935-2.228)^{*}\end{array}$ & 1.00 & $\begin{array}{l}2.245 \\
(0.741-6.804)\end{array}$ & 1.00 & $\begin{array}{l}0.673 \\
(0.412-1.098)\end{array}$ \\
\hline \multicolumn{11}{|l|}{ rs2249825 } \\
\hline $\mathrm{C}$ & 395 (76.1) & 85 (79.4) & $498(96.0)$ & 96 (89.7) & $271(52.2)$ & $49(45.8)$ & $504(97.1)$ & $102(95.3)$ & $359(69.4)$ & $73(68.2)$ \\
\hline G & $124(23.9)$ & 22 (20.6) & $21(4.0)$ & $11(10.3)$ & $248(47.8)$ & $58(54.2)$ & $15(2.9)$ & $5(4.7)$ & $158(30.6)$ & $34(31.8)$ \\
\hline OR $(95 \% \mathrm{CI})$ & 1 & $\begin{array}{l}0.824 \\
(0.495-1.374)\end{array}$ & 1.00 & $\begin{array}{l}2.717 \\
(1.269-5.819)^{*}\end{array}$ & 1.00 & $\begin{array}{l}1.293 \\
(0.8522-1.964)\end{array}$ & 1.00 & $\begin{array}{l}1.647 \\
(0.586-4.633)\end{array}$ & 1.00 & $\begin{array}{l}1.058 \\
(0 . .676-1.656)\end{array}$ \\
\hline AOR $(95 \%$ CI) & 1 & $\begin{array}{l}0.860 \\
(0.472-1.570)\end{array}$ & 1.00 & $\begin{array}{l}3.264 \\
(1.330-8.011)^{*}\end{array}$ & 1.00 & $\begin{array}{l}1.515 \\
(0.937-2.448)^{*}\end{array}$ & 1.00 & $\begin{array}{l}2.159 \\
(0.637-7.324)\end{array}$ & 1.00 & $\begin{array}{l}0.827 \\
(0.484-1.414)\end{array}$ \\
\hline \multicolumn{11}{|l|}{ rs1412125 } \\
\hline $\mathrm{T}$ & 358 (77.5) & $122(74.4)$ & 444 (96.1) & $150(91.5)$ & $236(51.1)$ & 84 (51.2) & 447 (96.8) & 159 (97) & $324(70.3)$ & $108(66.3)$ \\
\hline C & $104(22.5)$ & $42(25.6)$ & $18(3.9)$ & $14(8.5)$ & $226(48.9)$ & $80(48.8)$ & $15(3.2)$ & $5(3.0)$ & 137 (297) & 55 (33.7) \\
\hline OR (95\% CI) & 1 & $\begin{array}{l}1.185 \\
(0.784-1.791)\end{array}$ & 1.00 & $\begin{array}{l}2.302 \\
(1.118-4.742)^{*}\end{array}$ & 1.00 & $\begin{array}{l}0.995 \\
(0.696-1.420)\end{array}$ & 1.00 & $\begin{array}{l}0.937 \\
(0.335-2.620)\end{array}$ & 1.00 & $\begin{array}{l}1.204 \\
(0.823-1.763)\end{array}$ \\
\hline AOR $(95 \%$ CI) & 1 & $\begin{array}{l}1.370 \\
(0.841-2.231)\end{array}$ & 1.00 & $\begin{array}{l}2.702 \\
(1.181-6.182)^{*}\end{array}$ & 1.00 & $\begin{array}{l}1.086 \\
(0.721-1.636)\end{array}$ & 1.00 & $\begin{array}{l}1.145 \\
(0.365-3.592)\end{array}$ & 1.00 & $\begin{array}{l}1.170 \\
(0.741-1.847)\end{array}$ \\
\hline
\end{tabular}


HMGB1 = high-mobility group box protein $1 ; \mathrm{SNPs}=$ single nucleotide polymorphisms; $\mathrm{T} 2=$ tumor $>20 \mathrm{~mm}$ but $\leq 50 \mathrm{~mm}$ in greatest dimension; $\mathrm{N} 0=$ no regional lymph node metastasis; $\mathrm{N} 1$ = metastasis to movable ipsilateral level I, II axillary lymph node(s); N2 = metastases in ipsilateral level I, II axillary lymph nodes that are clinically fixed or matted or in clinically detected ipsilateral internal mammary nodes in the absence of clinically evident axillary lymph node metastasis; N3 = Metastases in ipsilateral infraclavicular (level III axillary) lymph node(s), with or without level I, II axillary node involvement, or in clinically detected ipsilateral internal mammary lymph node(s) and in the presence of clinically evident level I, II axillary lymph node metastasis; or metastasis in ipsilateral supraclavicular lymph node(s), with or without axillary or internal mammary lymph node involvement; $\mathrm{M} 0=$ no clinical or radiographic evidence of distant metastasis; $\mathrm{M} 1=$ distant detectable metastases as determined by classic clinical and radiographic means and/or histologically proven $>0.2 \mathrm{~mm}$; $\mathrm{G} 1=$ well differentiated; $\mathrm{G} 2$ = moderately differentiated; $\mathrm{G} 3$ = poorly differentiated.

a The odds ratios (ORs) and their corresponding 95\% confidence intervals (CIs) were estimated using logistic regression adjusted for age, tobacco and alcohol consumption.

* $p$ value $<0.05$ was considered statistically significant.

Table 4. Allele frequencies of 4 HMGBI SNPs in controls and patients with breast cancer.

\begin{tabular}{|c|c|c|c|c|c|c|c|}
\hline \multirow{2}{*}{$\begin{array}{l}\text { Allele } \\
\text { Variable }\end{array}$} & \multicolumn{2}{|c|}{ Luminal A + Luminal B } & \multicolumn{5}{|c|}{ HER2 + TNBC } \\
\hline & $\begin{array}{l}\text { Controls }(n=434) \\
\text { N }(\%)\end{array}$ & $\begin{array}{l}\text { Patients }(n=438) \\
N(\%)\end{array}$ & OR $(95 \% \mathrm{CI})$ & $\operatorname{AOR}(95 \% \mathrm{CI})$ & $\begin{array}{l}\text { Patients }(n=188) \\
N(\%)\end{array}$ & OR $(95 \% \mathrm{CI})$ & $\operatorname{AOR}(95 \% \mathrm{CI})$ \\
\hline \multicolumn{8}{|l|}{ rs1360485 } \\
\hline A & 333 (76.7) & $339(77.4)$ & 1.00 & $0.963(0.702-1.320)$ & $142(75.5)$ & 1.00 & $1.068(0.716-1.594)$ \\
\hline G & $101(23.3)$ & $99(22.6)$ & 1.00 & $0.948(0.683-1.318)$ & $46(24.5)$ & 1.00 & $1.022(0.677-1.542)$ \\
\hline \multicolumn{8}{|l|}{ rs1045411 } \\
\hline G & $339(78.1)$ & $343(78.3)$ & 1.00 & $0.988(0.717-1.363)$ & 147 (78.2) & 1.00 & $0.995(0.658-1.506)$ \\
\hline A & $95(21.9)$ & $95(21.7)$ & 1.00 & $0.971(0.695-1.358)$ & $41(21.8)$ & 1.00 & $0.953(0.623-1.459)$ \\
\hline \multicolumn{8}{|l|}{ rs2249825 } \\
\hline C & $374(86.2)$ & $365(83.3)$ & 1.00 & $1.247(0.860-1.806)$ & 154 (81.9) & 1.00 & $1.376(0.868-2.181)$ \\
\hline G & $60(13.8)$ & $73(16.7)$ & 1.00 & $1.188(0.808-1.747)$ & $34(18.1)$ & 1.00 & $1.284(0.799-2.062)$ \\
\hline \multicolumn{8}{|l|}{ rs1412125 } \\
\hline $\mathrm{T}$ & $334(77.0)$ & $321(73.3)$ & 1.00 & $1.217(0.895-1.656)$ & $141(75)$ & 1.00 & $1.113(0.747-1.659)$ \\
\hline C & $100(23.0)$ & $117(26.7)$ & 1.00 & 1.208 (0.876-1.667) & $47(25)$ & 1.00 & $1.095(0.726-1.652)$ \\
\hline
\end{tabular}

The odds ratios (ORs) and their corresponding $95 \%$ confidence intervals (CIs) were estimated using logistic regression models. AOR $=$ adjusted odds ratio.

* $p$ value $<0.05$ was considered statistically significant.

Table 5. Allele frequencies of 4 HMGBI SNPs and their association with clinical status in patients with breast cancer.

\begin{tabular}{|c|c|c|c|c|c|c|c|c|c|c|c|c|c|c|c|}
\hline \multirow{3}{*}{$\begin{array}{l}\text { Gene } \\
\text { Alle } \\
\end{array}$} & \multicolumn{15}{|c|}{ HER2 + TNBC (N=188) n (\%) } \\
\hline & \multicolumn{3}{|c|}{ Clinical Stage } & \multicolumn{3}{|c|}{ Tumor size } & \multicolumn{3}{|c|}{ Lymph node metastasis } & \multicolumn{3}{|c|}{ Distant metastasis } & \multicolumn{3}{|c|}{ Pathological grade } \\
\hline & $\begin{array}{l}\text { Stage } \\
\text { I/II }\end{array}$ & $\begin{array}{l}\text { Stage } \\
\text { III/IV }\end{array}$ & OR $(95 \% \mathrm{CI})$ & $\leqq \mathrm{T} 2$ & $>\mathrm{T} 2$ & OR $(95 \% \mathrm{CI})$ & N0+N1 & $\mathrm{N} 2+\mathrm{N} 3$ & OR $(95 \% \mathrm{CI})$ & M0 & M1 & OR $(95 \% \mathrm{CI})$ & $\mathrm{G} 1+\mathrm{G} 2$ & G3 & OR $(95 \% \mathrm{CI})$ \\
\hline \multicolumn{16}{|c|}{ rs1360485 } \\
\hline A & $107(75.4)$ & $31(67.4)$ & $\begin{array}{l}1.00 \\
\text { (reference) }\end{array}$ & $\begin{array}{l}135 \\
(95.1)\end{array}$ & $\begin{array}{l}35 \\
(76.1)\end{array}$ & 1.00 (reference) & $\begin{array}{l}69 \\
(48.6)\end{array}$ & $\begin{array}{l}19 \\
(41.3)\end{array}$ & $\begin{array}{l}1.00 \\
\text { (reference) }\end{array}$ & $\begin{array}{l}137 \\
(96.5)\end{array}$ & $\begin{array}{l}39 \\
(84.8)\end{array}$ & 1.00 (reference) & $\begin{array}{l}57 \\
(40.1)\end{array}$ & $\begin{array}{l}19 \\
(41.3)\end{array}$ & $\begin{array}{l}1.00 \\
\text { (reference) }\end{array}$ \\
\hline G & 35 (24.6) & 15 (32.6) & $\begin{array}{l}1.48 \\
(0.72-3.06)\end{array}$ & $7(4.9)$ & $\begin{array}{l}11 \\
(23.9)\end{array}$ & $\begin{array}{l}6.06 \\
(2.19-16.77)^{*}\end{array}$ & $\begin{array}{l}73 \\
(51.4)\end{array}$ & $\begin{array}{l}27 \\
(58.7)\end{array}$ & $\begin{array}{l}1.34 \\
(0.69-2.63)\end{array}$ & $5(3.5)$ & $7(15.2)$ & $\begin{array}{l}4.92 \\
(1.48-16.35)^{*}\end{array}$ & $\begin{array}{l}85 \\
(59.9)\end{array}$ & $\begin{array}{l}27 \\
(58.7)\end{array}$ & $0.95(0.49-1.87)$ \\
\hline \multicolumn{16}{|c|}{ rs1045411 } \\
\hline G & $109(74.1)$ & 29 (70.7) & $\begin{array}{l}1.00 \\
\text { (reference) }\end{array}$ & $\begin{array}{l}137 \\
(93.2)\end{array}$ & $\begin{array}{l}33 \\
(80.5)\end{array}$ & 1.00 (reference) & $\begin{array}{l}73 \\
(49.7)\end{array}$ & $\begin{array}{l}15 \\
(36.6)\end{array}$ & $\begin{array}{l}1.00 \\
\text { (reference) }\end{array}$ & $\begin{array}{l}142 \\
(96.6)\end{array}$ & $\begin{array}{l}34 \\
(82.9)\end{array}$ & 1.00 (reference) & $\begin{array}{l}58 \\
(39.5)\end{array}$ & $\begin{array}{l}18 \\
(43.9)\end{array}$ & $\begin{array}{l}1.00 \\
\text { (reference) }\end{array}$ \\
\hline A & $38(25.9)$ & $12(29.3)$ & $\begin{array}{l}1.19 \\
(0.55-2.56)\end{array}$ & $10(6.8)$ & $8(19.5)$ & $3.32(1.22-9.07)^{*}$ & $\begin{array}{l}74 \\
(50.3)\end{array}$ & $\begin{array}{l}26 \\
(63.4)\end{array}$ & $\begin{array}{l}1.71 \\
(0.84-3.49)\end{array}$ & $5(3.4)$ & $7(17.1)$ & $\begin{array}{l}5.85 \\
(1.75-19.55)^{*}\end{array}$ & $\begin{array}{l}89 \\
(60.5)\end{array}$ & $\begin{array}{l}23 \\
(56.1)\end{array}$ & $0.83(0.41-1.68)$ \\
\hline \multicolumn{16}{|c|}{ rs2249825 } \\
\hline C & $115(74.7)$ & $23(67.6)$ & $\begin{array}{l}1.00 \\
\text { (reference) }\end{array}$ & $\begin{array}{l}145 \\
(94.2)\end{array}$ & $\begin{array}{l}25 \\
(73.5)\end{array}$ & 1.00 (reference) & $\begin{array}{l}77 \\
(50.0)\end{array}$ & $\begin{array}{l}11 \\
(32.4)\end{array}$ & $\begin{array}{l}1.00 \\
\text { (reference) }\end{array}$ & $\begin{array}{l}146 \\
(94.8)\end{array}$ & $\begin{array}{l}30 \\
(88.2)\end{array}$ & 1.00 (reference) & $\begin{array}{l}61 \\
(39.6)\end{array}$ & $\begin{array}{l}15 \\
(44.1)\end{array}$ & $\begin{array}{l}1.00 \\
\text { (reference) }\end{array}$ \\
\hline G & $39(25.3)$ & $11(32.4)$ & $\begin{array}{l}1.41 \\
(0.63-3.16)\end{array}$ & $9(5.8)$ & $9(26.5)$ & $\begin{array}{l}5.80 \\
(2.10-16.03)^{*}\end{array}$ & $\begin{array}{l}77 \\
(50.0)\end{array}$ & $\begin{array}{l}23 \\
(67.6)\end{array}$ & $\begin{array}{l}2.09 \\
(0.95-4.58)\end{array}$ & $8(5.2)$ & $4(11.8)$ & $2.43(0.69-8.60)$ & $\begin{array}{l}93 \\
(60.4)\end{array}$ & $\begin{array}{l}19 \\
(17.0)\end{array}$ & $0.83(0.39-1.76)$ \\
\hline \multicolumn{16}{|c|}{ rs1412125 } \\
\hline $\mathrm{T}$ & $105(74.5)$ & $33(70.2)$ & $\begin{array}{l}1.00 \\
\text { (reference) }\end{array}$ & $\begin{array}{l}134 \\
(95.0)\end{array}$ & $\begin{array}{l}36 \\
(76.6)\end{array}$ & 1.00 (reference) & $\begin{array}{l}62 \\
(44.0)\end{array}$ & $\begin{array}{l}26 \\
(55.3)\end{array}$ & $\begin{array}{l}1.00 \\
\text { (reference) }\end{array}$ & $\begin{array}{l}132 \\
(93.6)\end{array}$ & $\begin{array}{l}44 \\
(93.6)\end{array}$ & 1.00 (reference) & $\begin{array}{l}63 \\
(44.7)\end{array}$ & $\begin{array}{l}13 \\
(27.7)\end{array}$ & $\begin{array}{l}1.00 \\
\text { (reference) }\end{array}$ \\
\hline C & $36(25.5)$ & $12(29.8)$ & $1.24(0.6-2.57)$ & $7(5.0)$ & $\begin{array}{l}11 \\
(23.4)\end{array}$ & $\begin{array}{l}5.85 \\
(2.12-16.17)^{*}\end{array}$ & $\begin{array}{l}79 \\
(56.0)\end{array}$ & $\begin{array}{l}21 \\
(44.7)\end{array}$ & $\begin{array}{l}0.63 \\
(0.33-1.23)\end{array}$ & $9(6.4)$ & $3(6.4)$ & $1.00(0.26-3.86)$ & $\begin{array}{l}78 \\
(55.3)\end{array}$ & $\begin{array}{l}34 \\
(72.3)\end{array}$ & $\begin{array}{l}2.11 \\
(1.03-4.34)^{*}\end{array}$ \\
\hline
\end{tabular}

The odds ratios (ORs) and their corresponding 95\% confidence intervals (CIs) were estimated using logistic regression models, age, tobacco and alcohol consumption.

$\mathrm{SNP}=$ single nucleotide polymorphism; HMGB1 $=$ high-mobility group box protein 1 ; HER2 $=$ human epidermal growth factor receptor 2 ; $\mathrm{TNBC}=$ triple-negative breast cancer; $\mathrm{T} 2=$ tumor $>20 \mathrm{~mm}$ but $550 \mathrm{~mm}$ in greatest dimension; $\mathrm{N0}=$ no regional lymph node metastasis; $\mathrm{N} 1=$ metastasis to movable ipsilateral level $\mathrm{I}, \mathrm{II}$ axillary lymph node(s); $\mathrm{N} 2=$ metastases in ipsilateral level I, II axillary lymph nodes that are clinically fixed or matted or in clinically detected ipsilateral internal mammary nodes in the absence of clinically evident axillary lymph node metastasis; N3 = Metastases in ipsilateral infraclavicular (level III axillary) lymph node(s), with or without level I, II axillary node involvement, or in clinically detected ipsilateral internal mammary lymph node(s) and in the presence of clinically evident level I, II axillary lymph node metastasis; or metastasis in ipsilateral supraclavicular lymph node(s), with or without axillary or internal mammary lymph node involvement; $\mathrm{M} 0=$ no clinical or radiographic evidence of distant metastasis; $\mathrm{M} 1=$ distant detectable metastases as determined by classic clinical and radiographic means and/or histologically proven $>0.2 \mathrm{~mm} ; \mathrm{G} 1=$ well differentiated; $\mathrm{G} 2=$ moderately differentiated; $\mathrm{G} 3=$ poorly differentiated .

${ }^{*} p$ value $<0.05$ was considered statistically significant.

Breast cancer is the most commonly diagnosed neoplasm and the third leading cause of cancer-associated mortality in the United States, with 22.2 mortalities per 100,000 women associated with breast cancer each year [29]. The 5-year relative survival rate for breast cancer has gradually increased since the early 1990s; between 2007 and 2011 it was $\sim 89.2 \%$ [29]. The prognosis of patients with breast cancer is critically dependent on the disease stage at the time of diagnosis. Therefore, it is important to increase screening rates and genetic testing for hereditary breast cancer, to increase the chances of early diagnosis $[30,31]$. The current study is the first to examine the distribution of the rs1360485, 
rs1045411, rs2249825 and rs1412125 SNPs and their possible association with breast cancer development. We also investigated the associations of these HMGB1 SNPs with clinical status, clinical pathologic markers, and susceptibility for breast cancer. In analyses adjusted for potential confounding factors, there were no significant differences between cases and controls in regard to the frequency of rs1360485, rs1045411, rs2249825 and rs1412125 polymorphisms. However, the presence of one $\mathrm{G}$ allele in the rs1360485 SNP, one $\mathrm{G}$ allele in the rs2249825 SNP, or one C allele in the rs1412125 SNP increased the likelihood of developing $\mathrm{T} 2$ breast cancer. Moreover, having one $\mathrm{G}$ allele in the rs1360485 SNP, one A allele in the rs1045411 SNP, or one $\mathrm{G}$ allele in the rs2249825 SNP was associated with a higher likelihood of developing lymph node metastatic disease. These results indicate that HMGB1 SNPs contribute to tumor size and lymph node metastasis in breast cancer patients.

This study found that having one $G$ allele in the rs1360485 SNP or one G allele in the rs2249825 SNP increased the risk of developing T2 breast cancer and distant metastasis in HER2 and TNBC subtypes when compared with luminal A and luminal B subgroups. Similarly, having one $G$ allele in the rs 2249825 or one $C$ allele in the rs1412125 increases the risk of developing T2 breast cancer in HER2 and TNBC breast cancer subtypes. It is already established that overexpression of the HMGB1 gene is implicated in the development, invasion and metastasis of breast cancer [32]. In addition, HMGB1 is involved in the chemotherapeutic resistance of breast cancer cells [33, 34]. However, more research is required to determine whether an association exists among advanced-stage disease, HMGB1 expression levels and HMGB1 genotype, and clarification is needed in regard to the effects of the HMGB1 genotype on breast cancer risk.

In conclusion, our results demonstrate an association between HMGB1 gene variants and the risk of breast cancer. However, we dose not recruited the survival results of breast cancer patients. Future research could evaluate the association of HMGB1 polymorphisms with survival of breast cancer patients. We show that HMGB1 gene variants significantly increase the risk of developing T2 breast cancer and lymph node metastasis among Chinese Han females. This study indicates a correlation exists between HMGB1 polymorphisms and breast cancer risk. HMGB1 may therefore serve as a predictive marker for breast cancer therapy.

\section{Acknowledgments}

This work was supported by grant from the Medical and Health Science and Technology Project of Zhejiang Province (2012KYB230); Taichung Veterans
General Hospital (TCVGH-NTUST1068502 and TCVGH:1063701C)

\section{Competing Interests}

The authors have declared that no competing interest exists.

\section{References}

1. Jemal A, Bray F, Center MM, Ferlay J, Ward E, Forman D. Global cancer statistics. CA: a cancer journal for clinicians. 2011; 61: 69-90.

2. Amir E, Freedman OC, Seruga B, Evans DG. Assessing women at high risk of breast cancer: a review of risk assessment models. Journal of the National Cancer Institute. 2010; 102: 680-91.

3. Nagy R, Sweet K, Eng C. Highly penetrant hereditary cancer syndromes. Oncogene. 2004; 23: 6445-70.

4. Antoniou AC, Pharoah PD, Narod S, Risch HA, Eyfjord JE, Hopper JL, et al. Breast and ovarian cancer risks to carriers of the BRCA1 5382insC and 185delAG and BRCA2 6174delT mutations: a combined analysis of 22 population based studies. Journal of medical genetics. 2005; 42: 602-3.

5. Park HL, Ziogas A, Chang J, Desai B, Bessonova L, Garner C, et al. Novel polymorphisms in caspase- 8 are associated with breast cancer risk in the California Teachers Study. BMC cancer. 2016; 16: 14.

6. Mosevitsky MI, Novitskaya VA, Iogannsen MG, Zabezhinsky MA. Tissue specificity of nucleo-cytoplasmic distribution of HMG1 and HMG2 proteins and their probable functions. Eur J Biochem. 1989; 185: 303-10.

7. Bustin M, Lehn DA, Landsman D. Structural features of the HMG chromosomal proteins and their genes. Biochim Biophys Acta. 1990; 1049: 231-43.

8. Bustin M. Regulation of DNA-dependent activities by the functional motifs of the high-mobility-group chromosomal proteins. Mol Cell Biol. 1999; 19: 5237-46.

9. Martinotti S, Patrone M, Ranzato E. Emerging roles for HMGB1 protein in immunity, inflammation, and cancer. ImmunoTargets and therapy. 2015; 4: 101-9.

10. Wang LH, Wu MH, Chen PC, Su CM, Xu G, Huang CC, et al. Prognostic significance of high-mobility group box protein 1 genetic polymorphisms in rheumatoid arthritis disease outcome. International journal of medical sciences. 2017; 14: 1382-8.

11. Wang B, Yeh CB, Lein MY, Su CM, Yang SF, Liu YF, et al. Effects of HMGB1 Polymorphisms on the Susceptibility and Progression of Hepatocellular Carcinoma. International journal of medical sciences. 2016; 13: 304-9.

12. Hu W, Liu PY, Yang YC, Chen PC, Su CM, Chao CC, et al. Association of HMGB1 Gene Polymorphisms with Lung Cancer Susceptibility and Clinical Aspects. International journal of medical sciences. 2017; 14: 1197-202.

13. Wu $\mathrm{HH}$, Liu YF, Yang SF, Lin WL, Chen SC, Han CP, et al. Association of single-nucleotide polymorphisms of high-mobility group box 1 with susceptibility and clinicopathological characteristics of uterine cervical neoplasia in Taiwanese women. Tumour biology : the journal of the International Society for Oncodevelopmental Biology and Medicine. 2016.

14. Exner R, Sachet M, Arnold T, Zinn-Zinnenburg M, Michlmayr A, Dubsky P, et al. Prognostic value of HMGB1 in early breast cancer patients under neoadjuvant chemotherapy. Cancer medicine. 2016; 5: 2350-8.

15. Yue L, Zhang Q, He L, Zhang M, Dong J, Zhao D, et al. Genetic predisposition of six well-defined polymorphisms in HMGB1/RAGE pathway to breast cancer in a large Han Chinese population. Journal of cellular and molecular medicine. 2016; 20: 1966-73.

16. Elston CW, Ellis IO. Pathological prognostic factors in breast cancer. I. The value of histological grade in breast cancer: experience from a large study with long-term follow-up. Histopathology. 1991; 19: 403-10.

17. Wang CQ, Tang CH, Wang Y, Jin L, Wang Q, Li X, et al. FSCN1 gene polymorphisms: biomarkers for the development and progression of breast cancer. Scientific reports. 2017; 7: 15887

18. Wang CQ, Li Y, Huang BF, Zhao YM, Yuan H, Guo D, et al. EGFR conjunct FSCN1 as a Novel Therapeutic Strategy in Triple-Negative Breast Cancer. Scientific reports. 2017; 7: 15654

19. Wang CQ, Tang CH, Chang HT, Li XN, Zhao YM, Su CM, et al. Fascin-1 as a novel diagnostic marker of triple-negative breast cancer. Cancer medicine. 2016; 5: 1983-8.

20. Wang $Y, \mathrm{Li} X \mathrm{XP}$, Yin JY, Zhang $\mathrm{Y}, \mathrm{He} \mathrm{H}$, Qian $\mathrm{CY}$, et al. Association of HMGB1 and HMGB2 genetic polymorphisms with lung cancer chemotherapy response. Clinical and experimental pharmacology \& physiology. 2014; 41: 408-15.

21. Lin YJ, Ho TJ, Lin TH, Hsu WY, Huang SM, Liao CC, et al. P-coumaric acid regulates exon 12 splicing of the ATP7B gene by modulating hnRNP A1 protein expressions. Biomedicine (Taipei). 2015; 5: 10.

22. Li TC, Li CI, Liao LN, Liu CS, Yang CW, Lin CH, et al. Associations of EDNRA and EDN1 polymorphisms with carotid intima media thickness through interactions with gender, regular exercise, and obesity in subjects in Taiwan: Taichung Community Health Study (TCHS). Biomedicine (Taipei). 2015; 5: 8. 
23. Barrett JC, Fry B, Maller J, Daly MJ. Haploview: analysis and visualization of LD and haplotype maps. Bioinformatics. 2005; 21: 263-5.

24. Ohmori H, Luo Y, Kuniyasu H. Non-histone nuclear factor HMGB1 as a therapeutic target in colorectal cancer. Expert Opin Ther Targets. 2011; 15: 183-93.

25. Reismann M, Wehrmann F, Schukfeh N, Kuebler JF, Ure B, Gluer S. Carbon dioxide, hypoxia and low pH lead to overexpression of c-myc and HMGB-1 oncogenes in neuroblastoma cells. Eur J Pediatr Surg. 2009; 19: 224-7.

26. Ahn MY, Kwon SM, Cheong HH, Park JH, Lee J, Min SK, et al. Toll-like receptor 7 agonist, imiquimod, inhibits oral squamous carcinoma cells through apoptosis and necrosis. J Oral Pathol Med. 2012; 41: 540-6.

27. Lv W, Chen N, Lin Y, Ma H, Ruan Y, Li Z, et al. Macrophage migration inhibitory factor promotes breast cancer metastasis via activation of HMGB1/TLR4/NF kappa B axis. Cancer letters. 2016; 375: 245-55.

28. Dhumale SS, Waghela BN, Pathak C. Quercetin protects necrotic insult and promotes apoptosis by attenuating the expression of RAGE and its ligand HMGB1 in human breast adenocarcinoma cells. IUBMB life. 2015; 67: 361-73.

29. Torre LA, Bray F, Siegel RL, Ferlay J, Lortet-Tieulent J, Jemal A. Global cancer statistics, 2012. CA: a cancer journal for clinicians. 2015; 65: 87-108.

30. Wood DE. National Comprehensive Cancer Network (NCCN) Clinical Practice Guidelines for Lung Cancer Screening. Thoracic surgery clinics. 2015; 25: 185-97.

31. Moyer VA, Force USPST. Medications to decrease the risk for breast cancer in women: recommendations from the U.S. Preventive Services Task Force recommendation statement. Annals of internal medicine. 2013; 159: 698-708.

32. Ni P, Zhang Y, Liu Y, Lin X, Su X, Lu H, et al. HMGB1 silence could promote MCF-7 cell apoptosis and inhibit invasion and metastasis. International journal of clinical and experimental pathology. 2015; 8: 15940-6.

33. Apetoh L, Ghiringhelli F, Tesniere A, Criollo A, Ortiz C, Lidereau R, et al. The interaction between HMGB1 and TLR4 dictates the outcome of anticancer chemotherapy and radiotherapy. Immunological reviews. 2007; 220: 47-59.

34. Amornsupak K, Insawang T, Thuwajit P, P OC, Eccles SA, Thuwajit C. Cancer-associated fibroblasts induce high mobility group box 1 and contribute to resistance to doxorubicin in breast cancer cells. BMC cancer. 2014; 14: 955. 